\title{
Network Scheduling Model of Cloud Computing based on Particle Swarm Optimization Algorithm
}

\author{
$\mathrm{Ke} \mathrm{Lu}^{1}$ and Junxia Meng ${ }^{2}$ \\ ${ }^{1}$ Department of international education, Jiaozuo University, Jiaozuo, 454003, \\ China \\ ${ }^{2}$ College of Information Engineering, Jiaozuo University, Jiaozuo, 454003, \\ China \\ E-mail:Kelu126@163.com
}

\begin{abstract}
The paper proposed a network scheduling in cloud computing based on intelligence Particle Swarm Optimization algorithm aimed at the disadvantages of cloud computing network scheduling. Firstly, on the basis of cloud model, used intelligence Particle Swarm Optimization algorithm with strong ability of global searching to find the better solution of cloud computing network scheduling then turned the better solution into the initial pheromone of improved Particle Swarm Optimization algorithm, and found out the cloud computing network scheduling and the algorithm's global optimal solution through improved Particle Swarm Optimization information communications and feedbacks. Finally, made comparison test of the three benchmark function on the basis of MATLAB, the results showed, compared with traditional intelligence Particle Swarm Optimization algorithms, the improved algorithm can preferably allocate the resources in cloud computing model, the effect of prediction model time is more close to actual time, can efficiently limit the possibility of falling into local convergence, the optimal solution's time of objective function value is shorten which meet the user's needs more.
\end{abstract}

Keywords: Network Computing, Cloud Computing, Particle Swarm Optimization Algorithm, Network Scheduling

\section{Introduction}

In cloud computing, the allocation of resources is a very important issue, the unsatisfactory allocation of resources can easily led the cloud's servers crashed and other servers in idle. So in cloud environment, the problem mostly need to solve is the ways to control any server's resources allocation and use condition by the information communication of local and in the Internet to make better use of the resources. Literature [1] made researches of the resources allocation conditions in different environment. Literature [2] proposed the resources allocation mechanism of self-management, self-adjustment and self-protection. Literature [3-5] proposed a resources allocation system applies to extensive distributed system, which efficiently increased the system's service quality under cloud computing.

Cloud computing is a combination of parallel computing, distributed computing and virtual technology, a hot technology of nowadays computer industry. The cloud system firstly combined computer, storage device and so on and formed resources pool, then the users could choose the corresponding resources by their needs, this dynamically offers users a computing service environment with reliable and ensure quality of service(QOS). Network scheduling is one of the core technologies of cloud computing which has big effect on the whole performance of cloud computing [6]. 
Aimed at the network scheduling of cloud computing, a scholar came up with a scheduling algorithm of HADOOP, this algorithm scheduled by network priority and submission time, which was easy to realize but it ignored the difference in networks and made a long response time of the networks [7]. Later some scholars proposed the cloud computing network scheduling algorithm with optimal efficiency; the network scheduling algorithm based on cost-driven; the network scheduling algorithm based on trust-driven, those algorithms is flexible and better meet the needs of the users, but they all aimed at a certain object so it's limited in application. A big amount of researches showed, cloud computing used in multiple network scheduling is not only reliable, quality of service(QOS) ensured but also be fair in network allocation, cloud computing multiple network scheduling is a worldrecognized NP problem[8]. It's very complex to solve with exhaustive search method, with swarm intelligence algorithm became mature, in recent years, some scholars brought particle swarm optimal algorithm, genetic algorithm and ant colony optimization into the network scheduling of cloud computing, which has a good result[9-10].

This paper mainly in the following aspects as the development and innovative work:

(1) On the basis of cloud model, proposed the intelligence Particle Swarm Optimization algorithm, analyzes the position, strengths and weakness in objective function's quantization individuals of solving problems.

(2) Proposes the measurement of cloud model's physicochemical characteristics. Analyzes the formal cloud model's algorithm steps of onedimension theory optimization and proves that this algorithm's atomization factor can reflect the discrete degree of cloud drop's distribution with experiments, no matter how the cloud model entropy and excess entropy value be, if only the atomization factor being the same, the cloud drop's discrete degree of cloud model. Besides, expectation (EX) only affects the whole position of cloud drop distribution without affecting the cloud drop's discrete degree.

(3) Proposes the applications of improved Particle Swarm Optimization algorithm in cloud computing network scheduling. Starts with the need of resources allocation in cloud computing environment, be similar with intelligence Particle Swarm Optimization algorithm in resources allocation, this text improves the update of fluoresce in based on researches of intelligence Particle Swarm Optimization algorithm, makes it better adapt to the resources allocation in cloud computing, through simulation experiment, the improvement of this algorithm has certain effect, and sets a theory basis for further improve the system's resources allocation effect under cloud computing model.

\section{Description Process}

The cloud model is a transformation model uses linguistic values to express the uncertainty between a certain conception and its quantification expression, it fully combines fuzziness and randomness and forms the mapping between qualitative and quantification, shows in figure 1.

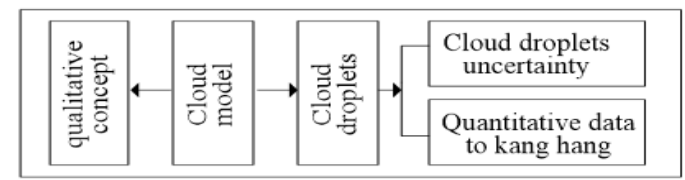

Figure 1. Transformation Schematic Of Cloud Mode's With Qualitative Concept 
Sets $U$ is discourse domain expressed by accurate numerical value, $A$ is corresponding qualitative concept in $\mathrm{U}$. If quantitative value $x \in u$ and $\mathrm{x}$ is a random implementation with likely normal distribution of qualitative concept $\mathrm{A}$ in discourse domain $\mathrm{U}$, the certainty degree $A(x) \in[0,1]$ of $\mathrm{x}$ to $\mathrm{A}$ is also a random number with likely normal distribution, then data array $\left(x, A\left(x_{i}\right)\right)$ is called as cloud drop, the whole element $x_{i}(i=1,2 \ldots n)$ in discourse domain $U$ and its certainty degree $A\left(x_{i}\right)$ for A, $i, e, \mathrm{n}$ data array $\left(x, A\left(x_{i}\right)\right)$, forms the cloud model with $\mathrm{n}$ cloud drop, calls $\mathrm{x}$ distribution in discourse domain $U$ as cloud distribution. The number characteristics of cloud model are expressed as expectation (Ex), entropy (En) and excess entropy $(\mathrm{He})$. Among them, expectation (Ex) refers to the central value of discourse domain $\mathrm{U}$, is the center of qualitative concept, reflects the cloud focus of the whole cloud drop swarm; entropy (En) refers to the range which can be received by fuzzy concept, $E n \succ 0$; excess entropy $(\mathrm{He})$ is a uncertain measurement of entropy, i,e, the excess entropy is the entropy's entropy, $\boldsymbol{H}_{e}>0$. The excess entropy reflects the degree of reach an agreement of cloud drop of representation qualitative concept or the concentration degree of cloud drop's representation qualitative concept; the bigger excess entropy is, the qualitative concept has worse common sense or the qualitative concept is worse decentralization.

\section{Camera parameters calibration based on Quantum Particle Swarm Optimization.}

Particle Swarm Optimization is a meta heuristic algorithm proposed in the inspired by the foraging behavior of birds [12]. Particle swarm optimization transforms the optimization problems which need to be solved into the foraging birds flying in the air, compare the particle in the algorithm to the birds in the population. The birds which are flying around for food in the model are just like the particle which are searching in the solution space, the position of one of the birds is a solution of the optimization problem to solve, and the food in the model is the optimal solution of the practical optimization problem [13]. Because the particle in the PSO could not avoid falling into local optimal, and if the parameter isn't fit in the operation process will cause the algorithm to produce the "premature" phenomenon, so the algorithm convergence is not up to the global minimum point and it has lower robustness [14]. Based on the PSO algorithm, researchers proposed quantum particle swarm optimization (QPSO) algorithm on the basis of Quantum mechanics theory $[15,16]$. In this paper, we use the improved quantum behaved particle swarm optimization algorithm to optimize the parameters of camera calibration.

\subsection{The Evolution Function}

The search speed and running position from $t$ to $t+1$ generations of the each particle in the particle swarm can be expressed as

$$
\left\{\begin{aligned}
x_{i d}(t+1) & =x_{i d}(t)+v_{i d}(t+1) \\
v_{i d}(t+1) & =v_{i d}(t)+c_{1} r_{1}\left(p_{i d}(t)-x_{i}(t)\right) \\
& +c_{2} r_{2}\left(p_{g d}(t)-x_{i d}(t)\right)
\end{aligned}\right.
$$

In the formula, $x_{i}=\left(x_{1}, x_{2}, \ldots ., x_{i d}\right)$ is the current position information of the ith particle, $v_{i}=\left(v_{1}, v_{2}, \ldots . . v_{i d}\right)$ is the current flight state speed information of the ith particle, $\mathrm{p}_{i}=\left(p_{1}, p_{2}, \ldots . . p_{i d}\right)$ is the best position of individual information of the ith particle, $p_{g}(t)$ is the global best position of particle swarm, $v_{i d}(t)$ and $v_{i d}(t+1)$ 
represents the speed of the ith particle in dth dimension of the th and $t+1$ th agent of iteration, $c_{1}$ and $c_{2}$ are the constant acceleration of particles, generallyc $c_{1}, c_{2} \varepsilon[0,2]$, $\mathrm{r}_{1} 、 \mathrm{r}_{2}$ are two arbitrary random numbers in section $(0,1)$.

Because the speed and position message of each particle in the quantum space appears in a certain probability, the particle state can be described by a wave function $\psi(x, t)$.

$$
\left\{\begin{array}{l}
|\psi|^{2} d x d y d z=Q d x d y d z \\
\left.\left|\int_{-\infty}^{+\infty}\right| \psi\right|^{2} d x d y d z=\int_{-\infty}^{+\infty} Q d x d y d z=1
\end{array}\right.
$$

According to the nature of DELTA function

$$
\left\{\begin{array}{l}
\delta(x-p)=\left\{\begin{array}{l}
\infty, x=p \\
0, x \neq p
\end{array}\right. \\
\left\{\int_{-\infty}^{+\infty} \delta(x-p) d x=1\right.
\end{array}\right.
$$

In the formula, $\delta$ is Dirac function, $p$ is the center of the Potential well of $\delta, \delta$ meet formula (13) and ensure the convergence behavior of quantum particles.

Each particle in the particle swarm meet Schrodinger equation.

$$
{ }_{i} \hbar \frac{\partial}{\partial t} \psi(x, t)=-\frac{\hbar^{2}}{2 m} \nabla^{2} \psi(x, t)+V(x) \psi(x, t)
$$

In the formula, $i$ is the imaginary unit, $h=h / 2 \pi$ is Planck constant, $V(x)=-\gamma \delta(x-p) V(x) \quad \gamma \neq 0$ is the potential energy of the particle in the trap motion space DELTA. The center of the space is $p . m$ is the mass of the particle. For the convenience of calculation, set not related to state and time variable of the particles, so particle behavior meet

$$
E \psi(x)=-\frac{\hbar^{2}}{2 m} \frac{d^{2} y}{d x^{2}} \psi(x)+V(x) \psi(x)
$$

In the formula, $E$ is the energy of a particle, $\Psi(x)$ is the steady state wave function.

Sort formula (5) we can get

$$
\frac{d^{2} \psi}{d x^{2}}+\frac{2 m}{\hbar^{2}}[E-V(x)]=0
$$

Do integral operation to the above formula

$$
\psi^{\prime}\left(0^{+}\right)-\psi^{\prime}\left(0^{-}\right)=-\frac{2 m \gamma}{\hbar^{2}} \psi(0)
$$

So, formula (6) is turned into

$$
\frac{d^{2} \psi}{d x^{2}}-\beta^{2} \psi=0, \beta=\sqrt{-2 m \hbar^{2}}(E<0)
$$

Substitute $V(x)=-\gamma \delta(x-p)$ into formula (5), we can get

$$
\psi(x-p)=\frac{1}{\sqrt{L}} e^{-|x-p| L}\left(L=\frac{1}{\beta}=\frac{\hbar^{2}}{m \gamma}\right)
$$

Make, $u=e^{-2|x-p| L}=\operatorname{rand}(0,1)$ then we get the evolutionary formula of QPSO 


$$
x=p \pm \frac{L}{2} \ln (1 / u)
$$

According formula (20) we get the evolutionary equation of QPSO

$$
\left\{\begin{array}{l}
x_{i}(t+1)=p_{i} \pm \beta \mid \text { mbest }-x_{i}(t) \mid \times \ln (1 / u) \\
p_{i}=\frac{a_{1} p_{i}+a_{2} p_{g}}{a_{1}+a_{2}} \\
\mid \text { mbest }=\frac{1}{N} \sum_{i=1}^{n} p_{i}
\end{array}\right.
$$

In the formula, $N$ is the total number of particles, $d$ is the particle dimension, $\mathrm{a} 1, \mathrm{a} 2, \mathrm{u} \varepsilon[0,1], p_{i}$ is the center of the potential well of the ith particle, mbest is the average of the all pbest of the population, $\beta$ is the contraction expansion factor which is controlling particle evolution speed.

\subsection{The Fitness Function}

Relate the camera parameters optimization calibration and QPSO by fitness function, express the camera calibration parameters as some factors of influence function, search for the best combination of influence by the evaluation of the fitness of each particle in the particle swarm to achieve the solution of optimization problems. In this article, all the camera parameters to be solved are optimization target, the particles in the population constituted by using the parameters to be optimized can be defined as

$$
\mathbf{x}=\left\{\begin{array}{l}
f, u_{0}, v_{0}, k_{1}, k_{2}, k_{3}, p_{1}, p_{2}, b_{1}, b_{2}, \\
\omega, \varphi, \kappa, t_{x}, t_{y}, t_{z}
\end{array}\right\}
$$

The fitness function $F$ can be described as

$$
F=\frac{1}{m} \sum_{i=1}^{m} \sqrt{\left[u_{i}-u_{i}^{\prime}(\mathrm{x})\right]^{2}+\left[v_{i}-v_{i}^{\prime}(\mathrm{x})\right]^{2}}
$$

In the formula, $m$ is the number of the calibration reference points, $\mu_{i}$ and $v_{i}$ is the pixel coordinates of the ith reference point, $\mu_{i}$ and $v_{i}$ is the estimated value calculated according to the camera model.

\subsection{Algorithm Implementation}

QPSO algorithm can effectively avoid falling into local optimal point, the algorithm realization steps are as follows:

(1) To calculate the internal parameters $(f, u 0, v 0)$ and the external parameters $\left(\varrho, \varphi, k, t_{x}, t_{y}, t_{z}\right)$ of the camera, at first, initialize the distortion parameters of camera which is $(k 1=k 2=k 3=p 1=p 2=b 1=b 2=0)$.

(2) Regard the initial value in step (1) as mean value, generate $n$ particles in gauss distribution randomly with appropriate mean square deviation, define the particle $x$ and fitness function $F$ as formula(12) and (13).

(3) Initialize the position message of all the particles of the population in the search space. Make $p_{i}(0)=x_{i}(0)$, and the initial global extreme $p_{g}(0)$ is the value of 
the particle which fitness is least in the generated particles. Suppose the iteration variable $t=0$, the particle generated in step (2) is the particles of 0th generation.

(4) Calculation the position $p_{i}(t)$ of the potential well $\delta$ according to formula (11), compare the fitness of $p_{i}(t)$ and $x_{i}(t+1)$, make $p_{i}(t+1)=\min \left\{p_{i}(t), x_{i}(t+1)\right.$ select the particle with the least fitness from the individual extreme $p_{i}(t+1)$ of the evolved $\mathrm{n}$ particles as the global extreme value $p_{g}(t+1)$. Then calculate mbest.

(5) The iteration variable $t$ increase 1. Judge if $t$ is bigger than the maximum number of iterations. If it is bigger, stop it, otherwise, turn back to (4).

(6) The global extreme $p_{g}$ achieved at last, is the final optimized value of camera parameter calibration with QPSO.

\section{Experimental Results}

In order to prove the performance of this algorithm, tests in two aspects, one is the performance of algorithm, the other is the network scheduling in cloud computing.

\subsection{Performance Test of Algorithm}

Makes comparison test using three benchmark function in literature and to test the algorithm's efficiency and performance. Using of MATLAB in Windows.

\section{Sphere function}

$$
f(x)=\sum_{i=1}^{m} x_{i}^{2}-100 \leq x_{i} \leq 100
$$

This is a continuous, un-modal convex function, the minimum point of the global function is zero, $i, e, x_{i}=0(i=1,2, \ldots n)$, there are on interaction among variables.

\section{Goldstein-Price function}

$$
\begin{aligned}
f(x)=\left[1+\left(x_{1}+x_{2}+1\right)^{2}+\left(18-13 x_{1}+2 x_{1}^{2}+5 x_{1} x_{2}+2 x_{2}^{2}\right)\right] \\
{\left[30+\left(2 x_{1}-3 x_{2}\right)^{2}\left(19-33 x_{1}+12 x_{1}^{2}+27 x_{2}^{2}\right)\right]-3 \leq 2, i=\{1,2\} }
\end{aligned}
$$

This is a multiple hump function, the minimum point of the whole function is $3, i, e, x_{i}=3(i=1,2, \ldots n)$, there are on interaction among variables.

\section{Ackely function}

$$
f(x)=-21 \exp \left(-0.2 \sqrt{\left.\left(\frac{1}{n} \sum_{i=1}^{m} \cos 2 \pi x_{i}\right)+21+r-34 \leq x_{i}\right)}\right.
$$

This is a multiple hump function with many local minimum points, the minimum point of the whole function is $0, i, e, x_{i}=0(i=1,2, \ldots n)$, there are on interaction among variables.

In the setting process of algorithm parameter, the scale of initial Particle Swarm Optimization is 500, iteration time is 200, parameter of fluoresce in is $p=0.6$, parameter of function is $\gamma=0.4$, initial fluoresce in is $l_{0}=10$.

Respectively tests 10 times of three functions and gets the best solution, worst solution and the average value. According to make compare with basic Particle Swarm Optimization algorithm, the results is as table 1, it is the convergence curve comparison between the algorithm in this text and intelligence Particle Swarm Optimization algorithm in three functions. 
Table 1. Comparison Of Testing Function

\begin{tabular}{|c|c|c|c|c|}
\hline function & algorithm & $\begin{array}{c}\text { the best } \\
\text { solution }\end{array}$ & $\begin{array}{c}\text { the worst } \\
\text { solution }\end{array}$ & average \\
\hline $\begin{array}{c}\text { Sphere } \\
\text { function }\end{array}$ & $\begin{array}{l}\text { intelligence } \\
\text { Particle Swarm } \\
\text { Optimization } \\
\text { algorithm }\end{array}$ & 0.0212501 & 0.0298742 & 0.0255600 \\
\cline { 2 - 5 } & $\begin{array}{l}\text { algorithm in } \\
\text { this text }\end{array}$ & 0.0019610 & 0.0105412 & 0.0062476 \\
\hline $\begin{array}{c}\text { Goldstein- } \\
\text { Price function }\end{array}$ & $\begin{array}{l}\text { intelligence } \\
\text { Particle Swarm } \\
\text { Optimization } \\
\text { algorithm }\end{array}$ & 3.0005126 & 3.0014521 & 3.0009822 \\
$\begin{array}{l}\text { algorithm in } \\
\text { this text }\end{array}$ & $\begin{array}{l}\text { intelligence } \\
\text { Particle Swarm } \\
\text { Optimization } \\
\text { algorithm }\end{array}$ & 3.0000003 & 3.0000301 & 3.0000112 \\
\cline { 2 - 5 } & $\begin{array}{l}\text { ACKELY } \\
\text { algorithm in } \\
\text { this text }\end{array}$ & 3.2456321 & 3.3562153 & 3.3109179 \\
\hline \multirow{2}{*}{\begin{tabular}{c} 
Aunction \\
\hline
\end{tabular}}
\end{tabular}

From table 1, the improved Particle Swarm Optimization algorithm is obviously better than intelligence Particle Swarm Optimization algorithm no matter in the best solution, the worst solution or the average. When basis intelligence Particle Swarm Optimization algorithm has certain iterations, it's easily fell into local optimum. This text efficiently limits the probability of felling into local optimum according to improve the fluoresce in of Particle Swarm Optimization, therefore reaches the optimum time of objective function.

\section{Network Scheduling of Improved Intelligence Particle Swarm Optimization Algorithm in Cloud Computing}

with the increasing number of sub-network, the algorithm in this text has certain advantages in network average transmission time and execution time. Mainly because the improved intelligence Particle Swarm Optimization algorithm can better distributes the resources in the model, prediction model time and effect is gradually close to actual time. As shown in figure 2

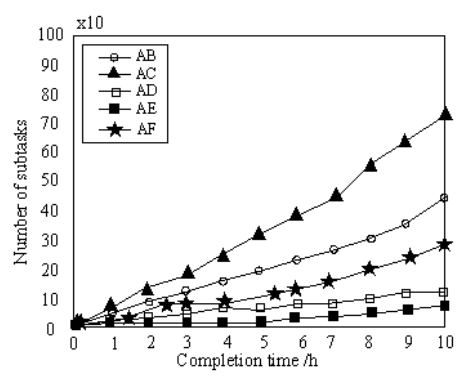

Figure 2. Network and Complementing Time of Intelligence Particle Swarm Optimization Algorithm 


\section{Conclusion}

How to make full use of the resources in cloud computing environment is a current-focusing problem. The method in this text improved the intelligence firefly algorithm in nature, combined the number of sub-task, resources with algorithm. In intelligence firefly algorithm, improved the method of firefly's fluoresce in position so as to make the firefly find the better object faster. On this improvement, the method reasonable solved the problem of balancing the network load and extending network, enhanced the global convergence of algorithm, it's valuable for increasing network operation. But there are still many practical problems in cloud computing to solve, resources distribution in cloud computing needs to be further researched.

\section{Acknowledgements}

The National Natural Science Foundation of China (U1204609); The Education Department of Henan Province Science and Technology Key Project (14A510011); The Youth Science Foundation of Henan Normal University (2012QK21)

\section{References}

[1] Y. Geng, J. He, H. Deng and K. Pahlavan, "Modeling the Effect of Human Body on TOA Ranging for Indoor Human Tracking with Wrist Mounted Sensor", 16th International Symposium on Wireless Personal Multimedia Communications (WPMC), Atlantic City, NJ, (2013) June.

[2] J. Fan, "The modified Levenberg-Marquardt method for nonlinear equations with cubic convergence", Math. Comput., vol. 81, pp. 447-466, (2012) June, http://dx.doi.org/10.1090/S0025-5718-2011-02496-8

[3] S. Li, Y. Geng, J. He and K. Pahlavan, "Analysis of Three-dimensional Maximum Likelihood Algorithm for Capsule Endoscopy Localization”, 2012 5th International Conference on Biomedical Engineering and Informatics (BMEI), Chongqing, China, (2012) October, pp. 721-725.

[4] E. Provenzi, C. Gatta, M. Fierro, et al., "A spatially variant white-patch and gray-world method for color image enhancement driven by local contrast", IEEE Transactions on Pattern Analysis and Machine Intelligence, vol. 30, no. 10, (2008), pp. 1757-1770.

[5] S. Zhou, G. Aggarwal and R. Chellapa, "Appearance characterization of linear lamebrain object, generalized photometric stereo and illumination- Invariant face recognition", IEEE Trans on PAMI, vol. 29, no. 2, (2007), pp. 230-245.

[6] A. Ali-Eldin, J. Tordsson and E. Elmroth, "Adaptive hybrid elasticity controller for cloud infrastructures", IEEE Network Operations and Management Symposium, (2012), pp. 204-212.

[7] D. Breitgand and A. Epstein, "SLA-aware Placement of Multi-Virtual Machine Elastic Services in Compute Clouds", IFIP/IEEE International Symposium on Integrated Network Management, Dublin, Ireland, (2011).

[8] W. -L Zhou, J. Xie and L. -B. Deng, "Sequencing Optimization Method for Drawing Train Operation Diagram of High-Speed Railway” Journal of Multimedia, vol. 8, no. 1, (2013), pp. 16-23.

[9] H. Chikara, K. Taketoshi, T. Norio, K. Shigeki and A. T. Motoshi, "Train stop deployment planning algorithm using a petri-net-based odeling approach quarterly", Report of RTRI, vol. 50, no. 1, (2009), pp. 8-13, http://dx.doi.org/10.2219/rtriqr.50.8 .

[10] K. P. Young, P. Seok and K. K. Joong, "Retinex method based on adaptive smoothing for illumination base on adaptive smoothing for illumination invariant face recognition", Signal Processing, vol. 88, (2008), pp. 1929-1945.

[11] S. Sudevalayam and P. Kulkami, "Affinity-Aware Modeling of CPU Usage for Provisioning Virtualized Applications", 2011 IEEE 4th International Conference on Cloud Computing, (2011), pp. 139-146 


\section{Authors}

Ke Lu, (Jiaozuo University, Jiaozuo, Henan, China, 454003) About the Author: Ke Lu, (1977-), male, department of international education, Jiaozuo University, China. Master Engineering. Research Field: computer network.

Junxia Meng, (College of Information Engineering, Jiaozuo

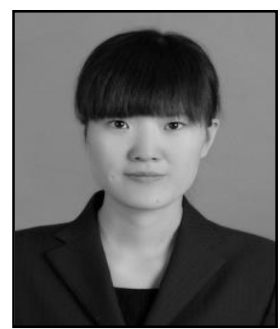

University, Jiaozuo 454003, China, About the Author: Meng Junxia, female, born in 1977. Master Engineering. Her main research interests include Computer software, and network security. 
International Journal of Grid Distribution Computing Vol. 8, No.4, (2015) 